\title{
A CAPACIDADE PARA O TRABALHO DA EQUIPE DE ENFERMAGEM INSERIDA NO AMBIENTE HOSPITALAR
}

\author{
THE ABILITY TO WORK OF THE NURSING STAFF INSERTED IN THE HOSPITAL ENVIROMENT
}

\section{Marcelly Santos Cossi $\mathrm{a}^{*}$, Raphael Raniere de Oliveira Costa $\mathrm{b}^{\mathrm{b}}$, Soraya Maria de Medeiros ${ }^{\mathrm{c}^{*}}$, Rejane Maria Paiva de Menezes ${ }^{\mathrm{d}^{*}}$}

amarcellycossi@gmail.com, braphaelraniere@hotmail.com, csorayamaria_ufrn@hotmail.com, drej@ufrnet.br

Data de entrada do artigo: 03/04/2014

Data de aceite do artigo: 15/08/2014

\section{RESUMO}

Introduçáo: Tendo em vista o desgastante trabalho desenvolvido pela equipe de enfermagem nos hospitais, torna-se essencial a avaliação da percepção desses trabalhadores sobre sua capacidade para o trabalho. Objetivo: O estudo tem por objetivo analisar os aspectos contextuais do índice de capacidade para o trabalho da equipe de enfermagem inserida no contexto hospitalar. Materiais e métodos: Utilizouse como metodologia a revisão de literatura, e os dados foram analisados segundo a análise contextual proposta por Hinds, Chaves e Cypress. Resultados: A maioria dos trabalhos científicos realizados entre os enfermeiros refere boa capacidade para o trabalho entre esses profissionais. Entretanto, ainda é considerável o número de trabalhadores que possui baixa ou moderada capacidade para o trabalho, sendo diversos os motivos organizacionais e pessoais responsáveis por essa realidade. Conclusóes: tornase necessário conhecer a condição de saúde dos trabalhadores e engajá-los em discussóes que busquem o desenvolvimento de programas voltados para a saúde do trabalhador.

Palavras-chave: enfermagem; saúde do trabalhador; enfermagem do trabalho.

\section{ABSTRACT}

Introduction: Given the exhausting work of the nursing staff in hospitals, it is essential to evaluate the perception of these workers on their ability to work. Objective: This article aims to analyze the contextual aspects of capability index for the work of the nursing staff in the hospital context. Materials and Methods: We used literature review as the methodology and the data was analyzed according to the contextual analysis proposed by Hinds, Chaves and Cypress. Results: Most of the scientific work among nurses regards to good work ability among these professionals. However, there is still a considerable number of workers who have low or moderate work ability, with various organizational and personal reasons that account for this reality. Conclusion: It is necessary to know the health condition of workers and engage them in discussions seeking to develop programs for the health of the worker.

Keywords: nursing; occupational health; occupational health nursing. 


\section{Introdução}

A equipe de enfermagem tem constante convivência com situações de dor e sofrimento vivenciadas pelos pacientes sob seus cuidados, o que contribui para o desenvolvimento de um misto de sentimentos no processo do cuidar, que pode ser agravado pela forma de organização do trabalho ${ }^{1}$.

A organização do trabalho no ambiente hospitalar é marcada por divisão fragmentada de tarefas, rígida estrutura hierárquica para o cumprimento das rotinas, dimensionamento inadequado de pessoal, resultando em elevados índices de absenteísmo e afastamento por doenças, além de desencadeamento de sintomas relacionados a burnout e insatisfação no trabalho ${ }^{2,3}$.

Diante da interação entre características do trabalho e recursos humanos, surge o processo denominado "capacidade para o trabalho", ação dinâmica que pode ser mantida ou restaurada se o devido cuidado com a saúde do trabalhador for tomado ${ }^{4}$.

A fim de avaliar a capacidade funcional dos trabalhadores, pesquisadores do Instituto de Saúde Ocupacional da Finlândia desenvolveram um questionário para determinar o índice de capacidade para o trabalho (ICT), identificando precocemente a perda da capacidade laboral e auxiliando na prevençáo de agravos, na manutenção da saúde dos trabalhadores e na melhoria da qualidade de vida no trabalho5.

O ICT analisa a percepção do trabalhador quanto às demandas físicas e mentais do trabalho, seu estado de saúde e sua capacidade funcional. $\mathrm{O}$ índice é calculado somando-se os pontos atribuídos para cada item, sofrendo uma variação de 7 a 49 pontos, que retratam a avaliaçáo do trabalhador sobre sua capacidade para o trabalhos.

Devido às características do trabalho desenvolvido pela equipe de enfermagem, como plantóes extensos, maior número de vínculos, além do trabalho doméstico que marca o gênero feminino, torna-se interessante estudar o ICT entre esses profissionais ${ }^{6}$.

Conscientes da importância de estudar as condições de trabalho da classe e identificar a precária situação desses trabalhadores, a Organização Internacional do Trabalho (OIT) juntamente com a Organização Mundial da Saúde (OMS) vêm estudando a profissão de enfermeiro ${ }^{7}$.

Em 1976, durante a 61 a Conferência da OIT, foi apresentado importante documento que tratava das condiçóes de vida, trabalho e emprego desses profissionais. Discutiram-se, na época, as condições insatisfatórias do trabalho de enfermagem relacionadas a fatores como extensas jornadas de trabalho, ausência de períodos de descanso, plantóes aos domingos e feriados sem justa compensação, períodos de trabalho incômodos ou fatigantes e o fato de a equipe de enfermagem não ser ouvida quanto ao planejamento e à tomada de decisóes acerca da prática profissional, do ensino e das condiçóes de trabalho ${ }^{7}$.

Tais dificuldades permanecem até os dias atuais, ou agravaram-se diante da crise socioeconômica e das modificaçóes do trabalho que interferem diretamente na saúde dos trabalhadores?

Considerando o exposto, é importante conhecer a realidade sobre a capacidade para o trabalho desses profissionais essenciais para o andamento do sistema de saúde. Logo, o presente artigo objetiva analisar os aspectos contextuais do índice de capacidade para o trabalho da equipe de enfermagem inserida no contexto hospitalar.

\section{Materiais e métodos}

Trata-se de um estudo teórico reflexivo que emergiu a partir das discussóes e reflexóes propostas pela disciplina intitulada "Bases filosóficas e teóricas de enfermagem na atenção a saúde", ofertada pelo Programa de PósGraduação em Enfermagem da Universidade Federal do Rio Grande do Norte, Brasil.

Buscando atender aos objetivos da pesquisa, utilizou-se o método de revisão de literatura, que se constitui de uma análise da produçâo bibliográfica em determinada área temática, dentro de um recorte de tempo, fornecendo uma visão geral ou um relatório do estado da arte sobre um tópico específico, corroborando novas ideias, métodos e subtemas que têm recebido destaque na literatura selecionada ${ }^{8}$.

A pesquisa bibliográfica abrangeu o período de 2005 a 2013. Os critérios de inclusão definidos para a seleção dos estudos foram: artigos completos; disponíveis nos idiomas português, inglês ou espanhol; e que atendessem o objetivo do estudo. Foram excluídos estudos no formato de teses, dissertaçóes, livros, carta ao editor, editorial e opinião de especialista, além de artigos indisponíveis no formato completo e artigos que não atendiam aos objetivos do estudo.

Para a organização dos resultados, utilizou-se como apoio a análise de contexto (AC) de Hinds, Chaves e Cypress9. A proposta de utilizar a análise de contexto como referencial surgiu do entendimento de que qualquer fenômeno ou problema sob estudo se insere em uma realidade que pode ter efeito significativo na sua solução, abrangendo dimensóes dotadas de significados e interaçóes intrínsecas e extrínsecas ao fenômeno estudado, subdividindo em quatro camadas interativas, a saber: contexto imediato, contexto específico, contexto geral e metacontexto. 
No contexto imediato, denominado "A capacidade para o trabalho da equipe de enfermagem", foi abordado como o índice de capacidade para o trabalho se apresenta entre a equipe de enfermagem. No contexto específico, "Ambiente laboral e suas implicaçóes", foram tecidas consideraçóes sobre o ambiente hospitalar em que a equipe de enfermagem está inserida, caracterizando o contexto específico. O contexto geral, "Aspectos subjetivos que permeiam o ICT", aborda as características individuais dos trabalhadores as quais podem contribuir para um ICT baixo ou elevado. Já no metacontexto, "ICT e suas soluçôes", elencam-se os aspectos que podem solucionar, melhorar ou manter o fenômeno em estudo.

\section{Desenvolvimento}

Observa-se no Quadro 1 o título dos artigos, objetivos e ano de publicação. Quanto ao ano de publicação, a maior frequência foi de publicaçóes referentes ao ano de 2012 (3). Os objetivos variaram, porém contemplam o objeto de estudo analisado.

\section{A Capacidade para o Trabalho da Equipe de Enfermagem}

Ao retratar o conceito do trabalhador sobre sua própria capacidade para o trabalho, observa-se que, apesar da realidade conhecida sobre o elevado índice de profissionais de enfermagem que se consideram incapazes para o trabalho, o estudo demonstrou haver uma mudança nesse sentido. A maioria dos trabalhos científicos realizados entre os enfermeiros refere boa capacidade para o trabalho entre esses profissionais.

Entretanto, ainda é considerável o número de trabalhadores que se julgam com baixa ou moderada capacidade para o trabalho, sendo diversos os motivos que podem ser responsáveis por essa realidade.

Estudo mostra que, entre os trabalhadores pesquisados, $387(83,2 \%)$ apresentaram boa capacidade para o trabalho e $78(16,8 \%)$, reduzida capacidade 5 . Outro estudo aponta que, entre os trabalhadores de enfermagem pesquisados, 43,3\% $(\mathrm{n}=216)$ dos trabalhadores possuíam baixa/moderada capacidade para o trabalho (reduzida capacidade) e 56,7\% ( $\mathrm{n}=282)$, boa/ótima capacidade ${ }^{10}$.

Quadro 1: Distribuição da frequência dos artigos científicos, segundo o objetivo, autoria, e ano de publicação.

\begin{tabular}{|c|c|c|}
\hline Artigo & Objetivo & Ano de publicaçáo \\
\hline $\begin{array}{l}\text { "Avaliação da capacidade funcional dos } \\
\text { trabalhadores de enfermagem" }\end{array}$ & $\begin{array}{l}\text { Avaliar a capacidade funcional dos trabalhadores de enfermagem de } \\
\text { um complexo hospitalar e sua relaçáo com características individuais } \\
\text { e de trabalho }\end{array}$ & 2005 \\
\hline $\begin{array}{l}\text { "Work-related stress, education and } \\
\text { work ability among hospital nurses" }\end{array}$ & $\begin{array}{l}\text { Determinar quais estressores estão presentes no ambiente de trabalho } \\
\text { dos enfermeiros; determinar se o nível educacional prediz a capacida- } \\
\text { de para o trabalho das enfermeiras }\end{array}$ & 2009 \\
\hline $\begin{array}{l}\text { "Work capacity evaluation among } \\
\text { nursing aides" }\end{array}$ & $\begin{array}{l}\text { Analisar a capacidade para o trabalho de auxiliares de enfermagem de } \\
\text { uma instituição pública de saúde }\end{array}$ & 2011 \\
\hline $\begin{array}{l}\text { "Fatores associados à capacidade para } \\
\text { o trabalho e percepçáo de fadiga em } \\
\text { trabalhadores de enfermagem da } \\
\text { Amazônia Ocidental" }\end{array}$ & $\begin{array}{l}\text { Analisar os fatores associados à capacidade inadequada para o traba- } \\
\text { lho e à percepçáo de fadiga entre os profissionais de enfermagem }\end{array}$ & 2011 \\
\hline $\begin{array}{l}\text { "Work ability among nursing per- } \\
\text { sonnel in public hospitals and health } \\
\text { centers in Campinas - Brazil" }\end{array}$ & $\begin{array}{c}\text { Avaliar a capacidade para o trabalho e aspectos de saúde do pessoal de } \\
\text { enfermagem }\end{array}$ & 2012 \\
\hline $\begin{array}{l}\text { "Perceived work ability and turnover } \\
\text { intentions: a prospective study among } \\
\text { Belgian healthcare workers" }\end{array}$ & $\begin{array}{c}\text { Explorar as relaçóes entre a capacidade para o trabalho dos enfer- } \\
\text { meiros e suas intençóes de deixar seu trabalho e profissão diante do } \\
\text { volume de tarefas exigidas }\end{array}$ & 2012 \\
\hline $\begin{array}{l}\text { "Intensidade da dor musculoesquelé- } \\
\text { tica e a (in) capacidade para o trabalho } \\
\text { na enfermagem" }\end{array}$ & $\begin{array}{c}\text { Avaliar a associação entre intensidade da dor musculoesquelética } \\
\text { e a redução da capacidade para o trabalho em trabalhadores de } \\
\text { enfermagem }\end{array}$ & 2012 \\
\hline
\end{tabular}


Corroborando os resultados apresentados, pesquisa realizada com equipe de enfermagem de quatro hospitais universitários da Croácia demonstrou que, dos 1.086 trabalhadores de enfermagem pesquisados, 380 tiveram baixa capacidade para o trabalho (WAI < 37), enquanto 706 apresentaram capacidade para o trabalho satisfatória (WAI $\ddagger 37)^{11}$.

Observa-se que, apesar de os resultados evidenciarem uma boa capacidade para o trabalho entre os trabalhadores de enfermagem, não se podem deixar de considerar os trabalhadores que apresentam características que resultam em uma baixa capacidade para o trabalho. E importante levar em conta as particularidades desses indivíduos, para que os motivos para tal problema sejam apontados e solucionados pelas instituiçóes empregadoras dos mesmos.

\section{Ambiente Laboral e suas Implicações}

A incapacidade para o trabalho pode ser atribuída às características do ambiente, relacionadas à forma de organização do trabalho. A organização laboral inadequada pode ser marcada pelo número insuficiente de profissionais no hospital para realizar as tarefas, pela realização de atividades repetitivas e monótonas, pela extensa jornada de trabalho, pelo elevado número de vínculos e pelo tempo de trabalho na instituição.

Em um estudo com 272 trabalhadores de enfermagem de um hospital de urgência e emergência de Rio Branco, foi observado que a jornada média semanal dos trabalhadores era de 63 horas. Logo, pode-se estabelecer um vínculo entre o aumento da jornada de trabalho em decorrência de outro emprego e a reduzida capacidade para o trabalho dos profissionais ${ }^{1}$.

Outro estudo apresentou um aspecto interessante em relação aos trabalhadores com dupla jornada de trabalho, pois possuíam ICT mais favorável do que aqueles com um único trabalho. É necessário conhecer os aspectos que permeiam essa situação e que contribuem para esse resultado destoante. Pode-se considerar que aspectos como a necessidade de complementação da renda, a perspectiva de um futuro financeiro melhor, o prazer gerado pelo trabalho, a possibilidade de trabalhar com pessoas, chefias e ambientes diferentes podem contribuir para uma melhor capacidade para o trabalho ${ }^{10}$.

\section{Aspectos Subjetivos que Permeiam o ICT}

As características pessoais dos trabalhadores também podem influenciar na determinação do índice de capacidade para o trabalho.
Sexo, idade, número de doenças ocupacionais, nível de escolaridade, prática de atividade física, índice de massa corporal (IMC) e número de filhos são aspectos pessoais que também contribuem para uma melhor ou pior capacidade para o trabalho.

Estudo aponta que o aumento do número de doenças pode ocasionar uma diminuição significativa da capacidade para o trabalho, sendo possível observar que a ocorrência de capacidade inadequada para o trabalho entre os indivíduos que referiram três ou mais doenças foi $48 \%$ maior em relação aos trabalhadores que não referiram a presença de doença ${ }^{1}$.

A prática de exercícios apresentou relação com a melhor capacidade para o trabalho, o que permite sugerir que sejam adotadas açóes que orientem a prática regular de uma atividade física pelos trabalhadores de enfermagem, a fim de melhorar suas condiçōes de saúde 5 .

Estudos apontaram ainda que indivíduos mais velhos apresentam uma reduzida capacidade para o trabalho, indicando a necessidade de se observar a capacidade laboral e sua relação com as condiçóes de trabalho, a fim de manter o trabalhador saudável enquanto permanecer no mercado de trabalho, prevenindo o envelhecimento precoce e a incapacidade laboral ${ }^{12}$.

Em relação à escolaridade, indivíduos com maior escolaridade apresentaram melhor índice de capacidade laboral quando comparados aos de menor escolaridade, o que expressa que, na divisão de trabalho da enfermagem, as tarefas consideradas pesadas são, na maioria das vezes, executadas pelos técnicos e auxiliares de enfermagem, contribuindo para um ICT mais baixo 5 .

Nesse contexto, o trabalho da enfermagem, em seus aspectos organizacionais e pessoais, é dotado de uma série de características que contribuem decisivamente para um decréscimo ou aumento na capacidade para o trabalho ${ }^{1}$.

\section{ICT e suas Soluções}

Os trabalhadores de enfermagem constituem uma categoria profissional submetida a um processo de trabalho que os expóe a uma série de riscos, elevando a probabilidade da ocorrência de agravos e doenças profissionais. Além disso, é um trabalho desgastante, marcado por uma série de estressores 5 .

Diante dessa realidade, a avaliação do ICT entre esses profissionais torna-se relevante para o acompanhamento da capacidade funcional, colaborando na intervenção e melhoria das condiçóes de saúde e trabalho. Além disso, fornece subsídios para a adoção de medidas que promovem a saúde e previnem agravos ${ }^{10}$.

O ICT pode tornar possíveis as intervenções de cunho individual e coletivo, de natureza ambiental e 
organizacional, que buscam restaurar e manter a capacidade para o trabalho e permitir melhorias nas condições de saúde e na qualidade de vida dessa classe trabalhadora. O estímulo à prática de atividades físicas e de lazer e a implementação de programas visando a preservação do sistema musculoesquelético são algumas açóes que podem contribuir para a manutenção da capacidade para o trabalho ${ }^{1,5}$.

Além de oferecer açóes que preservem a capacidade para o trabalho dos profissionais, torna-se necessária, para aliviar esse problema, a adoção de uma gerência participativa, a fim de aproximar o trabalhador das discussóes e do levantamento dos problemas no processo e na organização do trabalho, contribuindo para melhores soluções para os problemas ${ }^{10}$.

\section{Conclusões}

Este estudo, referente à análise contextual do fenômeno capacidade para o trabalho, contribuiu para a reflexão sobre os desafios presentes na operacionalização do trabalho de equipes de enfermagem que atuam no âmbito hospitalar, trabalho este fundamental na vida de todos os seres humanos que necessitam de cuidados nesse nível de atenção.

Através dos níveis interativos dos contextos analisados, foram identificados problemas de ordem técnica e pessoal, contribuindo para que a discussão se converta em açôes voltadas à busca de soluçóes que resultem na transformação dos processos de trabalho.

Observou-se que, diante da realidade do ambiente de trabalho que as instituiçóes hospitalares oferecem, marcado por uma série de riscos e estressores, como o sofrimento físico e psíquico, torna-se necessário conhecer individualmente a condição de saúde dos trabalhadores e engajá-los em discussões que busquem o desenvolvimento de programas voltados à saúde do trabalhador.

Além disso, estudos que contemplam a situação de saúde da equipe de enfermagem são vastos; entretanto, ainda são necessárias pesquisas que subsidiem a visão dos próprios trabalhadores em relação à sua capacidade para o trabalho, a fim de investir em soluçóes que melhorem as condiçóes de trabalho e a qualidade de vida dos profissionais.

Através do percurso metodológico adotado, foi possível conhecer os estudos dessa área que estão sendo publicados em periódicos nacionais e internacionais. $\mathrm{O}$ grande quantitativo de estudos indisponíveis gratuitamente ocasionou algumas limitaçōes, como, por exemplo, a restrição de acesso a estudos relevantes de diversas localidades do mundo, o que nos impede de fazer inferências mais concretas. Espera-se que as consideraçóes agregadas neste artigo possam ser apreciadas como um estímulo para a realização de novas investigações.

\section{Referências}

1. Vasconcelos SP, Fischer FM, Reis AOA, Moreno CRC. Fatores associados à capacidade para o trabalho e percepção de fadiga em trabalhadores de enfermagem da Amazônia ocidental. Rev Bras Epidemiol. 2011;14(4):688-97.

2. Batista AAV, Vieira MJ, Cardoso NCS, Carvalho GRP. Fatores de motivaçáo e insatisfaçáo no trabalho do enfermeiro. Rev Esc Enferm USP. 2005;39(1):85-91.

3. Avendaño C, Bustos P, Espinoza P, García F, Pierart T. Burnout y apoyo social en personal del servicio de psiquiatría de un hospital público. Ciencia y Enfermería 2009; 15(2):55-68.

4. Ilmarinen J, Tuomi K. Past present and future of work ability. In: Ilmarinen J, Lehtinen S. Past present and future of work ability. Helsinki: Finnish Institute of Occupational Health; 2004.

5. Raffone AM, Hennington EA. Avaliação da capacidade funcional dos trabalhadores de enfermagem. Rev Saúde Pública. 2005;39(4).

6. Silva JSHA, Ana GGV, Griep RH, Rotenberg L. Validade e confiabilidade do índice de capacidade para o trabalho (ICT) em trabalhadores de enfermagem. Cad Saúde Pública. 2011;27(6).

7. Fischer FM, Borges FNS, Rotenberg L, Latorre MRDO, Soares NS, Rosa PLFS, et al. Work ability of health care shift workers: what matters? Chronobiol Int. 2006;23:1165-79.

8. Noronha DP, Ferreira SMSP. Revisóes de literatura. In: Campello BS, Condón BV, Kremer JM, organizadores. Fontes de informação para pesquisadores e profissionais. Belo Horizonte: UFMG; 2000.

9. Hinds P, Chaves D, Cypress S. Context as a source of meaning and understanding. Qual Health Res. 1992;2(1):61-74.

10. Magnago TSBS, Lima ACS, Prochnow A, Ceron MS, Tavares JP, Urbanetto JS. Intensidade da dor musculoesquelética e a (in)capacidade para o trabalho na enfermagem. Rev Latino-Am Enfermagem. 2012;20(6):1125-33.

11. Golubic R, Milosevic M, Knezevic B, Mustajbegovic J. Work-related stress, education and work ability among hospital nurses. J Adv Nurs. 2009;65(11):2056-66.

12. Hilleshein EF, Souza LM, Lautert L, Paz AA, Catalan VM, Teixeira MG, Mello DB. Capacidade para o trabalho de enfermeiros de um hospital universitário. Rev Gaúcha Enferm. 2011;32(3):509-1. 